\title{
Indicadores biológicos de calidad de las aguas superficiales de la subcuenca del Río Viejo, utilizando Fitobentos (Diatomeas)
}

\author{
Silvia Hernández González \\ Centro para la Investigación en Recursos Acuáticos de Nicaragua, CIRA/UNAN-Managua. \\ silvia.hernandez@cira-unan.edu.ni
}

Recibido: 15 de octubre 2012 / Aceptado: 5 de diciembre 2012.

\section{RESUMEN}

El Río Viejo es afectado por las actividades agrícolas y ganaderas que se desarrollan en la subcuenca. De este se seleccionaron 15 sitios en el Río Viejo para evaluar la calidad de agua utilizando diatomeas como bioindicadores. Las muestras fueron tomadas en piedras y sedimento a lo largo de todo el río y tributarios. El objetivo principal del estudio fue determinar las especies más dominantes de diatomeas y su utilidad como indicadores biológicos. Según el índice de Shannon el $80 \%$ de los sitios muestreados presentaron contaminación moderada y un $20 \%$ se clasificó como aguas de intensa contaminación. Basado en el índice trófico de diatomeas - TDI (por sus siglas en inglés) (Kelly et al. 2001) para determinar el estado trófico, sus aguas se clasificaron como clases I, II, III y IV en la parte alta del río, sugiriendo un estado oligotrófico (Jordán y Breiera) a eutrófico; en la parte media se clasificó como I, IV y V; y en pate baja se reportaron clases II, III, IV y V, correspondiendo la última clasificación a los sitios El Cacao y Trinidad Casco Urbano como los puntos de mayor contaminación.

Palabras Claves: Fitobentos, diatomeas, índice, calidad de agua.

\section{INTRODUCCIÓN}

La necesidad de poder implementar métodos más rápidos y económicos para evaluar la calidad de agua del Río Viejo por su alto grado de degradación debido al desarrollo de las actividades agrícolas y ganaderas, conlleva a un estudio amplio y completo de su cuenca, incluyendo el uso de comunidades biológicas para valorar el estado de la calidad biológica de sus aguas. Para ello se utilizó a las diatomeas como bioindicadores, porque brindan información sobre la integridad biológica de un sistema acuático por su rápida respuesta a los cambios de las condiciones ambientales.

Por consiguiente, se realizó un estudio biológico a lo largo del río viejo, utilizándose a las diatomeas para valorar el grado de afectación que presentan sus aguas debido a las diferentes actividades que se desarrollan en la cuenca. Barbour (1999) considera que la riqueza de especies es un estimador del número de especies presentes en un área determinada y una alta riqueza sugiere una buena integridad biótica, dado que muchas se adaptan a las condiciones presentes del hábitat, tendiendo a disminuir con el incremento de la contaminación. En ese sentido el desarrollo de métodos para evaluar la calidad de las aguas es de amplio uso, siendo el TDI utlizado para evaluar el estado trófico.

\section{MATERIALES Y MÉTODOS}

El estudio se realizó en 15 sitios (de los cuales se tomaron 29 muestras) para diatomeas epilíticas (piedras) y epipsamicas (sedimento) en el Río Viejo y sus tributarios en 2010 y 2011. Las piedras fueron cepilladas suavemente para desprender las algas y transferidas a un frasco debidamente rotulado y fijada con alcohol al $4 \%$ para su transporte al laboratorio.

La materia orgánica de las algas fue eliminada por el método de peróxido de hidrógeno y montadas en placas permanentes con medio naphrax. La identificación de las taxa se realizó en microscopio compuesto laborlux y claves taxonómicas según Krammer \& Lange-Bertalot (1985), Kurt Krammer \& Lange-Bertalot (1988), Easter E. Cupp (1977) y Kurt Krammer \& Lange-Bertalot (1987). Para evaluar la calidad y estado trófico de agua, se utilizaron los índices de Shannon y Weaver y el índice trófico de diatomeas (TDI).

\section{RESULTADOS Y DISCUSIÓN}

La composición de diatomeas presentó un total de 99 taxa constituida principalmente por once géneros: Nitzschia (26), Navicula (21), Achnanthes (11), Gomphonema (6), Pinnularia (6), Cymbella (5), Cyclotella (3), Amphora (2), Aulacoseira (2), Fragilaria (2) y Rhopalodia (2), donde los tres primeros géneros se destacan por su mayor representación en cada una de las secciones de la subcuenca. 
Un género muy diverso fue Nitzschia que tiene afinidad a aguas salobres, orgánicamente contaminadas, ricas en nutrientes y pobres en óxigeno, donde N. palea se encontró en el $90 \%$ de todos los sitios muestreados, resultando ser abundante en varios sitios altamente contaminados por aguas residuales doméstica (Trinidad Casco Urbano: $48,1 \%$ ) y La Mojarra: $25 \%$ en el 2010; así como en los sitios Nacascolo $(27,4 \%)$, Santa Rosa $(30,9 \%)$, Trinidad Las Lajas $(41,5 \%)$ y El Coloncito $(20,2 \%)$ del 2011. Esto es reportado por muchos autores como característica de ambientes ricos en materia orgánica (Lange-Bertalo, 1979; Gómez, N. 1995; Kelly \& Whitton, 1995 y Van Dam, H. et al., 1994), siendo utilizada como indicadora de aguas fuertemente contaminadas por su alta tolerancia a la contaminación (Lange-Bertalot H. 1980).

Otro género diverso fue Navicula, donde N. mutica mostró registros abundantes en el punto El Cacao (46,1\%: 2010 y 97\%: 2011) característico por sus fuertes corrientes, porque recibe las aguas provenientes de la planta hidroeléctrica hidrogesa. Dicha especie se reporta para condiciones de alta conductividad, altas concentraciones de nutrientes y corrientes de flujos rápidos (Ana L. Martínez de Fabricius, et al., 2003), siendo las concentraciones de nutrientes para este estudio (PT: 0.15 mg.1-1 2010; PT: 0.137 mg.l-N-NH4: 5.75 mg.l-1 2011) y N. goeppertiana con dominancia en Abravieja de (82,3\%: 2011), donde también la velocidad de la corriente en este tramo del río es fuerte, turbulenta y con altas concentraciones de nutrientes (PT: 0.122 mg.l- 1; N$\mathrm{NH}_{4}$ : 6.08 mg.l-1).

Un tercer género fue Achnanthes el cual es común en un amplio rango de condiciones ambientales, siendo representado mayormente por la especie $A$. minuttissima en la parte alta (El Jordán: $48,3 \%$ ) y baja (El Cacao: 24,2\%) en 2010; (Jordán: 19.82\%) y Achnanthes biosolettiana (El Jordán: 20.7\%; La Breiera: 34\%) en 2011. A. minutissima es considerada una especie cosmopolita y reportada para aguas menos contaminadas. Descy (1979) la clasificó como sensitiva a la contaminación, sin embargo, Mayama, S. \& Kobayasi, H. (1984), la encontró en aguas de fuerte a severamente contaminadas, sin observarse altas densidades en aguas excesivamente contaminadas. Igualmente $A$. biosolettiana indica bajo nivel de contaminación.

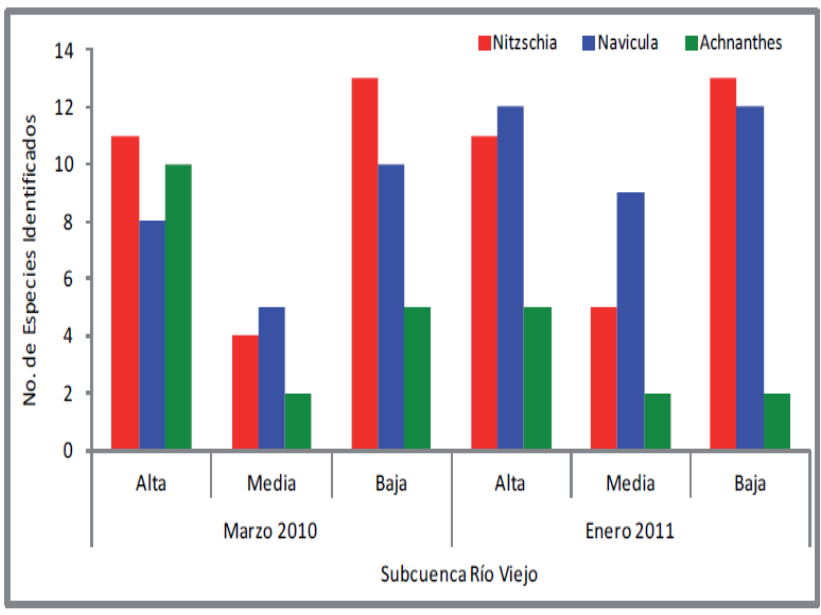

Figura 1. Géneros de diatomeas con mayor aporte a la riqueza de especies en cada una de las partes de la subcuenca del Río Viejo en ambos muestreos

Un hallazgo relevante fue la dominancia de Fragilaria construens en Nacascolo $(84,6 \%)$, Trinidad Las Lajas $(93,6 \%)$ y La Perla (55,3\%) 2010. En estos tres sitios las concentraciones de PT fueron 0.075 mg.l-1, $0.091 \mathrm{mg} .1-1$ y $0.121 \mathrm{mg} .1-1$ respectivamente, es de suponer que existe el efecto de una variable que favorece el desarrollo de dicha especie. Sin embargo Sabater, S. \& F. Sabater (1987) encontraron a esta especie muy abundante en zonas altamente contaminadas.

Otro género reportado fue Gomphonema sp, el cual se reportó solamente en Santa Rosa: 35,2\% (2010) La Breiera: 45,9\% y Los Encuentros: 53,7\% (2011), y la especie Cocconeis placentula, considerada cosmopolita y presente en condiciones de aguas limpias a moderadamente enriquecidas (Biggs, B. J. F. \& C. Kilroy, 2000), reportada en la parte baja (Los Calpules: 33,5\%) y media (Trinidad Las Lajas: $30,2 \%$ ) del 2010 y baja (Los Calpules: $36,2 \%$ y a Perla: 73,7\%) del 2011.

\section{Calidad de agua}

Los resultados obtenidos de la aplicación del índice Shannon (Figura 2) fueron relativamente bajos $\left(\mathrm{H}^{\prime}=\right.$ 0,15 - 2,61 bits). Según la clasificación de los distintos grados de contaminación (Wilhm, J. L. \& T. C. Dorris, 1968), el $80 \%$ de los sitios presentaron contaminación moderada y un $20 \%$ registró valores $<1$, clasificándose como aguas de intensa contaminación correspondiendoles los menores 
valores a Trinidad las lajas (0.36 bit) y el Cacao (0.15bit).

Basado en el Índice Trófico de Diatomeas (TDI por sus siglas en inglés) Kelly, M. G. et al. (2001), los sitios ubicados en la parte alta del río fueron clasificados como clases I, II, III y IV correspondiendoles un estado trófico de oligotrófico a eutrófico revelando un estado ecológico de excelente hasta pobre; los dos sitios de la parte media se clasificaron como clase IV y V (Trinidad Casco urbano) lo que sugiere un estado eutrófico a hipertrófico refiriendo un estado ecológico pobre a malo y clase I y V (Trinidad Las Lajas), indicando un estado ecológico excelente para el primer muestreo y pobre para el segundo; por último en la parte baja se reportaron clases II, III, IV y V, mostrando un estado trófico de oligo mesotrofico a hipereutrófico, refierendo un estado ecológico del agua de regular hasta mala. De todos los sitios El Cacao mostró ser el más altamente contaminado correspondiendole un estado trófico herpereutrófico en segundo muestreo.

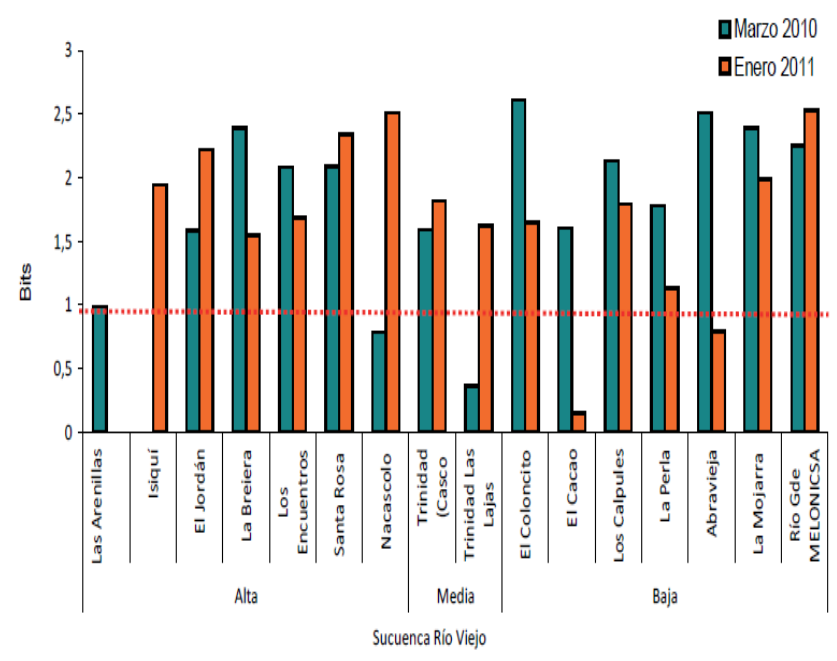

Figura 2. Índice de Diversidad de Shannon \& Weaver

Tabla 1. Rangos del indice TDI y su respectivo estado trófico, ecológico y clase (fiente: E. Szczepocka, B. Szule).

\begin{tabular}{|c|c|c|c|}
\hline TDI & Estado Trófico & Estado ecológico & Clase \\
\hline$<35$ & Oligotrófico & Excelente & I \\
\hline $35-50$ & 0ligo/mesotrófico & Buena & II \\
\hline $50-60$ & mesotrófico & Regular & III \\
\hline $60-75$ & eutrófico & Pobre & IV \\
\hline$>75$ & hipereutrófico & Mala & V \\
\hline
\end{tabular}

Tabla 2. Clasificación de la calidad de agua del río viejo utilizando el indice TDI.

\begin{tabular}{|c|c|c|c|}
\hline & \multicolumn{3}{|c|}{ TDI } \\
\hline & Sitios & Marzo, 2010 & Enero, 2011 \\
\hline \multirow{6}{*}{ Parte alta } & El Jordan & 48 (II) & 49 (II) \\
\hline & La Breiera & 30 (I) & 38 (II) \\
\hline & Los Encuentros & 54 (III) & 53 (III) \\
\hline & Santa Rosa & 72 (IV) & 71 (IV) \\
\hline & Nacascolo & 31 (I) & 71 (IV) \\
\hline & Isiqui & & 20 (I) \\
\hline \multirow[t]{2}{*}{ Parte media } & \begin{tabular}{|ll} 
Trinidad & Casco \\
Urbano & \\
\end{tabular} & $85(\mathrm{~V})$ & 74 (IV) \\
\hline & \begin{tabular}{|ll} 
Trinidad Las \\
Lajas
\end{tabular} & 27 (I) & 70 (IV) \\
\hline \multirow{7}{*}{ Parte baja } & El Coloncito & 52 (III) & 54 (III) \\
\hline & El Cacao & 73 (IV) & $99(\mathrm{~V})$ \\
\hline & Los Calpules & 68 (IV) & 59 (III) \\
\hline & La Perla & 48 (II) & 53 (III) \\
\hline & Abravieja & 62 (IV) & 69 (IV) \\
\hline & La Mojarra & 68 (IV) & 75 (IV) \\
\hline & MELONICSA & 53 (III) & 36 (II) \\
\hline
\end{tabular}

\section{CONCLUSIONES}

Los géneros con mayor representación a la riqueza de especies fueron: Nitzschia, Navicula y Achnanthes, donde los dos primeros tienen afinidad a aguas salobres, orgánicamente contaminadas, ricas en nutrientes y pobres en óxigeno, que los hace ser tolerantes a medios contaminados. Aunque Achnanthes es reportada para medios menos contaminados, se observó en sitios de bajo a fuerte contaminación.

La dominancia de Navicula mutica en El Cacao y en los dos muestreos (2010: 46,1\%; 2011: 97\%), indica medios con elevadas concentraciones del nutrientes principalmente fósforo y nitrógeno y fuerte flujo de la corriente.

Nitzschia palea, fue la especie de mayor importancia, por resultar frecuente en el $90 \%$ del total de sitios ubicados en toda la subcuenca, encontrándose en condiciones mesotróficas a eutróficas.

Los valores obtenidos de la aplicación del Indice de Shannon fueron relativamente bajos $\left(\mathrm{H}^{\prime}=0,15-\right.$ 2,61 ), señalando que el $80 \%$ de los sitios reportan contaminación moderada y solamente el $20 \%$ registra aguas de contaminación intensa.

En base al Índice Trófico de Diatomeas (TDI) los sitios muestreados en el Río Viejo presentaron un 
estado trófico de $(14 \%)$ oligotrófico, $(17 \%)$ oligo/mesotrófico, (24\%) mesotrófico, (38\%) eutrófico y $(7 \%)$ hipereutrófico.

\section{RECOMENDACIONES}

Considerando que los sitios evaluados se encuentran en su mayor porcentaje entre un estado mesotrófico a eutrófico lo que indica un estado ecológico pobre a regular, se requiere una programa de monitoreo que permita la implementación de un plan de gestión ambiental.

Desde el punto de vista biológico es necesario aumentar el número de puntos a valorar por subcuenca e identificar las diferentes fuentes de contaminación puntual que existan en el área.

\section{BIBLIOGRAFÍA}

Ana L. Martínez de Fabricius, N. Maidana, N. Gómez \& S. 4,* Sabater. 2003. Distribution patterns of benthic diatoms in a Pampean river exposed to seasonal floods: the Cuarto River (Argentina). Biodiversity and Conservation 12: 2443-2454, 2003.

Barbour, M.T., J. Gerritsen, B.D. Snyder, \& J.B. Stribling. 1999. Rapid Bioassessment protocols for Use in Streams and Wadeable Rivers: Periphyton, Benthic Macroinvertebrates and Fish, Second Edition. EPA 841-B-99-002. U.S. Environmental Protection

Agency; Office of Water; Washington, D.C.

Biggs, B. J. F. \& C. Kilroy. 2000. Stream Periphyton Monitoring Manual.

Descy, J. P. 1979. A new approach to Water Quality Estimation using Diatoms. Nova HedwigiaBeiheft 64: 305-323.

Gómez, N. 1998. Use of epipelic diatoms for evaluation of water quality in the matanzaRiachueleo (Argentina) a Pampean Plain river.

Kelly M. G. et al. 2001. The Trophic Diatom Index A User, s Manual. Revised edition. R.\& De Technical Report E2/TR2, Bristol: Environment Agency.
Kelly, M. G. and Whittton B. A. 1995. The trophic Diatom Index: a new index for monitoring eutrophication in rivers. J appl. Phycol. 7: 433-444.

Lange-Bertalot H. 1980. Fur taxonomischen Revision einiger okologischer wichtiger Navicula lineolatae Cleve. Die Formenkreise um Navicula lanceolata, Navicula viridula und Navicula cari. Cryptogamie Algologie 1: 2950 .

Mayama, S. \& Kobayosi, H. 1984. The separated Distribution of the Two Varieties of Achnanthes minutissima Kuetz. According to the Degreee of River Water Pollution.

Sabater, S. \& F. Sabater (1987). Comunidades de diatomeas en localidades altamentes contaminadas del Ter (Cataluña, Ne España). Limnetica 3: 103-109.

Van Dam, H. et al. 1994. A coded checklist and ecological indicator values of freshwater diatoms from the netherlands.

Wilhm, j. L. \& T. C. Dorris, 1968. Biological parameters for Water Quality Criteria. Bioscience. 18: 77 - 481 